\title{
REAL LIFE EGYPTIAN EXPERIENCE OF COMBINATION THERAPY (SIMEPREVIR/SOFOSBUVIR) IN EXPERIENCED NON CIRRHOTIC HCV PATIENTS \\ BY
}

\author{
Engy A. Wahsh ${ }^{1}$, Amal K. Hussein ${ }^{2}$, Ahmed A. Gomaa ${ }^{3}$
}

FROM

${ }^{1}$ Assistant lecturer of Clinical Pharmacy department, Faculty of Pharmacy, Nahda University, Beni Suef, Egypt.

${ }^{2}$ Assistant Professor of Pharmaceutical Technology department, Faculty of Pharmacy, Minia University, Minia, Egypt.

${ }^{3}$ Assistant Professor of Tropical Medicine Department, Faculty of Medicine, Fayoum University, Fayoum, Egypt

\section{Abstract}

\section{Background}

Egypt has the highest prevalent in presence of chronic HCV infection that may lead to cirrhosis, portal hypertension, hepatocellular failure and hepatocellular carcinoma. Developing of new drugs in HCV treatment has been developed using direct acting antiviral activity. In this study we assess the safety and efficacy of a combination therapy Sofosbuvir/Simprevir in the treatment of chronic experienced HCV

\section{Method}

Sixty five chronic HCV patients were recruited from the outpatient clinic of Tropical Medicine Department at Fayoum public hospital. A combination of Sofosbuvir (400 mg) and Simeprevir (150 mg) was administered for those patients once daily over a period of 12 weeks. All patients have been followed up for clinical and laboratory parameters and HCV PCR to evaluate the efficacy and safety of this therapy.

\section{Result}

Our results show high Sustained Virologic Response rate (SVR12) which was 98.5\% (64/65). Majority of patients (49.45\%) reported adverse events (AEs) during treatment and most common AEs were headache, fatigue, pruritus, dizziness and photosensitivity.

\section{Conclusion}

On the basis of the evidence currently available, it seems fair to suggest that the combination therapy of (SMV/SOF) in the treatment of chronic HCV genotype IV experienced patient who are non-cirrhotic is safe and effective. This was evidenced by monitoring patients' clinically and using hepatic parameters after drug administration. The adverse events affected patients were mild and tolerable among patients.

Keywords Chronic hepatitis C, genotype IV, simeprevir 


\section{Introduction}

Chronic infection with HCV is a worldwide health problem which may result in cirrhosis, portal hypertension, hepatocellular failure and carcinoma. Prevalence of HCV infection was the highest in Egypt it was 15\%. The wide spread of HCV in Egypt was primarily attributed to the parenteral antischistosomal therapy by Intravenous tartar emetic which was used from 1950 s to the 1980 s to control the wide pool of schistosomiasis in Egypt (Hanno et al., 2016).

The dangers of exposure to human blood were unappreciated; as a result, $24.3 \%$ of Egyptians who received that therapy got infected with HCV. The absence of disposable needles and syringes 50 years ago while giving these parenteral medication was the main reason that Led to HCV positive cases in about $20.6 \%$ of users. Additionally, nosocomial infections from medical procedures and household transmission is considered to be a risk factor (Gomaa et al., 2017).

Genotype 4 is considered the most prevalent one in Egypt (approximately 90\%). For many years, the standard of care therapy was a combination of pegylated interferon and Ribavirin for 48 weeks with response rates that do not exceed $60 \%$ and limited by both eligibility and tolerability. For this reason, genotype $4 \mathrm{HCV}$ has been considered to be difficult to treat. New treatment approaches were displayed after the rapid development of medical intervention by potent active direct acting antiviral agents (DAAs) that help us to reach a sustained virological response (SVR) that exceeds $90 \%$ nowadays (Gaetano, 2014).

The most commonly used therapy in Egypt for chronic HCV before discovery of direct-acting antiviral drugs was pegylated-interferon alfa (PEG-IFN) administered with ribavirin, with efficacy demonstrated as a sustained virological response (SVR) more than $60 \%$ of patients after 48 weeks of therapy. A new era in HCV treatment has developed using direct acting antiviral drugs DAA which lead to increase of virologic response reach to 95\% in different genotypes (Press, 2015).

The first approved DAA drug by the food and drug administration FDA was Sofosbuvir (SOF) a nucleotide analogue HCV NS5B polymerase inhibitor which has a high tolerability and preferable pharmacokinetic profile. Among the newly developed drugs was the second-generation non-structural 3/4A (NS3/4A) protease inhibitor simeprevir (SMV). Both drugs administered orally once daily and have potent antiviral activity with wide genotypic coverage (Sulkowski et al., 2014).

The aim of our study is to assess the safety and efficacy of combination therapy Sofosbuvir/Simprevir in the treatment of chronic HCV genotype IV non-cirrhotic experienced patients.

\section{Patients and methods}

Sixty five chronic HCV patients were recruited from the outpatient clinic at Tropical Medicine Department, Fayoum public hospital, from February 2016 to November 2016, with a follow-up period of 36 weeks, according to the following inclusion and exclusion criteria

\section{Inclusion Criteria:}

Egyptian patients aged $18-70$ years, have Detectable serum quantitative HCV RNA who are treatment experienced non cirrhotic patients. 
Exclusion criteria: any of the following,

- Naive patients

- Cirrhotic patients

- Patients with Direct serum bilirubin $>2 \mathrm{mg} / \mathrm{dl}$.

- Serum albumin $<2.8 \mathrm{~g} / \mathrm{dl}$.

- $\quad$ INR $\geq 1.7$

- Platelet count $<50000 / \mathrm{mm} 3$

- Hepatocellular carcinoma (HCC), except 4 weeks after intervention aiming at cure with no evidence of activity by dynamic imaging (CT or MRI).

- Serum creatinine $>2.5 \mathrm{mg} / \mathrm{dL}$. If creatinine is between 1.5 and $2.5 \mathrm{mg} / \mathrm{dL}$, eGFR should be calculated and should exceed $30 \mathrm{~mL} / \mathrm{min}$ with favorable nephrological consultation.

- Extra-hepatic malignancy except after two years of disease-free interval.

- Co-infection with hepatitis B virus or HIV

- Pregnancy or inability to use effective contraception

- Organ transplant.

\section{Study design:}

The study was conducted on HCV genotype 4 patients who were non-cirrhotic treatment experienced. All patients received Sofosbuvir (SOF) $400 \mathrm{mg}$ and simeprevir (SMV) $150 \mathrm{mg}$ (SMV/SOF) once daily for 12 weeks.

All patients have been followed up after receiving the therapy and data was collected about the following parameters with the purpose of comparing the changes before and after receiving the combination therapy: Full clinical examination and history taking information were also recorded.

1. Laboratory investigations include:

- Complete blood count

- Complete biochemical profile: aspartate amino transferase (AST), alanine amino transferase (ALT), serum albumin, serum creatinine, total bilirubin, prothrombin concentration and international normalized ratio (INR).

- Serum alpha-fetoprotein (AFP) was also measured before treatment (if > 100 triphasic CT abdomen should be done to exclude HCC).

- Thyroid function test

- Random blood sugar

2. Follow up for clinical, laboratory assessment and HCV PCR for the purpose of documenting the patients' response (efficacy) and side effects (safety) of treatment.

The end point was defined as (SVR) of less than 15 IU per milliliter at 12 weeks (SVR 12) and 24 weeks(SVR 24) of treatment determined by quantitative PCR for HCV. 


\section{Statistical analyses}

Descriptive analysis has been performed and frequency and percentage of all variables' categories have been tabulated. For continuous data, the mean and standard deviation (Mean \pm SD) have been reported. Statistical associations between pairs of categorical variables were assessed using $\chi 2$-tests. Changes in the measured laboratory parameters before and three months after the treatment have been examined using dependent student-t test to determine whether improvement or deterioration in laboratory investigations has been observed. P-values less than 0.05 were considered as significant results. All statistical analyses were performed using IBM-SPSS version 22.0 (SPSS, Chicago, IL, USA).

\section{Results}

Sixty five treatment experienced non cirrhotic patients were subjected to receive combination therapy SMV/SOV for treatment of chronic HCV. There were 82\% (53 of 65) males patients in the study group. In table 1 we present the baseline demographic and clinical characteristics of patients

Table 1: Patient Demographics and Baseline Characteristics $(n=65)$ :

\begin{tabular}{|c|c||}
\hline Title & M \pm SD $; \mathrm{N}(\%)$ \\
\hline Age (years) & $44.18 \pm 10.02$ \\
\hline Gender & $53(82 \%)$ \\
Male & $12(18 \%)$ \\
Female & $29.64 \pm 5.93$ \\
\hline BMI & $20(9 \%)$ \\
\hline Comorbidities & $7(10.7 \%)$ \\
\hline DM & $6(9.2 \%)$ \\
\hline HTN & 0 \\
\hline Smokers & 0 \\
\hline HBsAg positive & \\
\hline Liver cirrhosis & \\
\hline
\end{tabular}

The results show that a serum HCV RNA decline was observed with most of patients. Sustained Virologic Response rate (SVR12) in this study population was 98.5\% (64/65) (primary end point), and the same reached at second end point (SVR 24).

As shown in table 2, all patients in the study noticed before treatment and three months later. A significant decline in the liver enzyme value after 3 months of treatment as ALT before treatment was $49.45 \pm 27.12$ and after treatment was $22.69 \pm 9.42$ while AST was $43.27 \pm 19.64$ and $23.5 \pm 8.39$ before and after treatment respectively. A 
significant increase in albumin, alpha fetoprotein and glucose level were observed in the study. There was no significant difference in other values.

Table 2: Laboratory data for patients

\begin{tabular}{||c|c|c|c||}
\hline & Before ttt (65) & 3 months(65) & p-value \\
\hline HCV RNA (IU) log 10 & $5.31 \pm 0.82$ & $1.18 \pm 0.00$ & $<0.001$ \\
\hline ALT (IU/L) (ULN: 4 0 IU/L) & $49.45 \pm 27.12$ & $22.69 \pm 9.42$ & $<0.001$ \\
\hline AST (IU/L) (ULN: 40 IU/L) & $43.27 \pm 19.64$ & $23.5 \pm 8.39$ & $<0.001$ \\
\hline AFP (IU/L) (ULN: 10 IU/L) & $19.4 \pm 30.3$ & $15.8 \pm 28.8$ & 0.001 \\
\hline Albumin (g/dL) & $4.01 \pm 0.52$ & $4.2 \pm 0.5$ & 0.021 \\
\hline Total Bilirubin (mg/dL) & $0.89 \pm 0.42$ & $0.86 \pm 0.33$ & 0.582 \\
\hline WBC $\times 103 / m m 3$ & $6.40 \pm 2.02$ & $7.06 \pm 1.60$ & 0.359 \\
\hline Platelets $\times 103 / \mathrm{mm} 3$ & $187.8 \pm 62.67$ & $200.2 \pm 56.58$ & 0.129 \\
\hline HGB (G/L) & $14 \pm 1.8$ & $13.8 \pm 1.95$ & 0.503 \\
\hline$*$ PC (\%) & $87 \%$ & & \\
\hline$*$ INR & $1.13 \pm 0.12$ & & 0.422 \\
\hline Creatinine (mg/dL) & $0.88 \pm 0.27$ & $0.97 \pm 0.37$ & 0.056 \\
\hline Glucose (mg/dL) & $101.73 \pm 21.94$ & $111.5 \pm 34.2$ & \\
\hline$*$ TSH & $2.04 \pm 2.15$ & & \\
\hline$*$ values measured at beginning of treatment only & \\
\hline
\end{tabular}

According to results shown in table $3,46.15 \%$ patients reported adverse events (AEs) during treatment. The most common AEs reported were headache, fatigue, pruritus, and photosensitivity. No Serious AEs were reported. Only $7.7 \%$ of patients report increase in bilirubin which considered as an AE. All patients complete the study period.

Table 3: Summary of adverse events and laboratory abnormalities

\begin{tabular}{||c|c||}
\hline Title & $\mathrm{N}(\%)$ \\
\hline No. (\%) of patients with any adverse event, $\mathrm{n}$ & $30(46.15 \%)$ \\
\hline No. (\%) of patients with a serious adverse event & 0 \\
\hline Fatigue & $12(18.5 \%)$ \\
\hline Photosensitivity & $8(12.3 \%)$ \\
\hline Headache & $18(27.7 \%)$ \\
\hline Pruritis & $1(1.5 \%)$ \\
\hline Arthralgia & $2(3.1 \%)$ \\
\hline Fever & $3(4.6 \%)$ \\
\hline Bilirubin increase $>1.2$ & $5(7.7 \%)$ \\
\hline
\end{tabular}




\section{Discussion}

This study aim to assess the efficacy of combination therapy simeprevir plus sofosbuvir in treatment of HCV experienced patients who are non-cirrhotic. Our observation show that the efficacy of this regimen for treatment chronic infection with HCV as the overall SVR12 was 98.5\%. As shown in several studies our results approximately reliable with the foremost publication which has efficacy in treatment of HCV using the same combination.

Our findings are matched with a recent study conducted on 6211 patients and SVR12 was $97.6 \%$ in all patients. Difficult to treat group show significantly decrease in SVR12 when compared to easy group as SVR12 were 93\% and 96\% respectively after 12 weeks(Eletreby et al. 2016). Also a Phase III (PLUTO) found that the SVR was $100 \&$ among study subjects after administration of Simeprevir in combination with sofosbuvir in treatment of naïve and experienced patients with and without compensated cirrhosis (Buti et al., 2017).

Our results were matched with the OSIRIS study, where the SVR12 rate attained was also $100 \%$ for patients with and without cirrhosis who received the same combination for 12 weeks (El Raziky et al., 2015). Similar results have also been reported by Moreno et al.,2015 with 100\% SVR12 of HCV genotype 4-infected patients with cirrhosis, treated with or without ribavirin (Bourgeois et al., 2017). The results of this study is complemented by Phase III OPTIMIST-1 study were the patient achieved 97\% SVR12 in HCV genotype 1-infected patients without cirrhosis treated with simeprevir plus sofosbuvir for 12 weeks achieved 97\% SVR12 (Kwo et al., 2016), while in Phase III OPTIMIST-2 study in patients with cirrhosis achieved 83\% SVR12 (Lawitz et al., 2016).

Our result in a good agreement with previous study in which HCV-1 infected patients treated with sofosbuvir and ledipasvir for 12 weeks have achieved $97 \%$ and 99\% SVR12 with and without ribavirin respectively (Gitlin et al., 2014). The results of this study are concur with other study conducted by El- khayat et al., 2016 who assessed SVR 12 for 583 Egyptian patients with HCV genotype 4 infection with overall SVR12 rate in their study was $(95.7 \%)$, after receiving SIM/SOF without ribavirin for 12 weeks and was lower among cirrhotic patients (80.8\%) (El-Khayat et al., 2016).

The target SVR12 achieved in our study was higher than that achieved by Flamm et al., 2014 who studied the effect of Ledipasvir (NS5A inhibitor) plus sofosbuvir for treating HCV-4 infected patients, Patients achieved SVR12 of 87\% when RBV was added to this combination for 12 weeks in HCV genotype 1 and 4 infected patients with decompensated cirrhosis (Flamm et al., 2014).

We can conclude that oral regimen of simeprevir/sofosbuvir combination is an effective and well tolerated regimen for patients with chronic experienced non cirrhotic HCV genotype 4, and it has better SVR

\section{REFERENCES}

Bourgeois S., Negrin S., Gustot T.,and Francque S., (2017). Sofosbuvir in combination with simeprevir $+/$ - ribavirin in genotype 4 hepatitis $\mathrm{C}$ patients with advanced fibrosis or cirrhosis: real-life experience from Belgium. PloS one, 12(1), p.56. 
Buti M., Calleja J., and Lens S.,(2017). Simeprevir in combination with sofosbuvir in treatment-naïve and -experienced patients with hepatitis C virus genotype 4 infection: a Phase III, open-label, single-arm study (PLUTO). Alimentary pharmacology \& therapeutics, 45(3), pp.468-475.

Eletreby R., Elakel W., and Said M.,(2016). Real life Egyptian experience of efficacy and safety of Simeprevir/Sofosbuvir therapy in 6211 chronic HCV genotype IV infected patients. Liver international: official journal of the International Association for the Study of the Liver, (May), pp.1-8.

El Raziky M., Gamil M.,and Hammad R., (2015). Treatment of hepatitis C genotype 4 patients with simeprevir and sofosbuvir: preliminary results from a phase IIa, partially randomised, open-label trial conducted in Egypt (OSIRIS). Hepatology, 62, p.784A.

El-Khayat H., Fouad Y., and Maher M., (2016). Efficacy and safety of sofosbuvir plus simeprevir therapy in Egyptian patients with chronic hepatitis C: a realworld experience. Gut,5,325-332.

Flamm S., Everson G., and Charlton M., (2014). Ledipasvir / Sofosbuvir With Ribavirin for the Treatment of HCV in Patients With Decompensated Cirrhosis : Preliminary Results of a Prospective Multicenter Study. Annual Meeting of the American Association for the Study of Liver Diseases,1-18

Gaetano J.,( 2014). Benefit - risk assessment of new and emerging treatments for hepatitis $\mathrm{C}$ : focus on simeprevir and sofosbuvir. Drug, Healthcare and Patient Safety, 6, pp.37-45.

Gitlin N., Puoti M.,and Symonds W.,( 2014). Ledipasvir and Sofosbuvir for Untreated HCV Genotype 1 Infection., pp.1889-1898.

Gomaa A., Allam N.,and Kassas M., (2017). Hepatitis C infection in Egypt: prevalence, impact and management strategies. Hepatic Medicine: Evidence and Research, 9, pp.17-25.

Hanno A., Elwazzan D., and Hafez R., (2016). A Real Life Study on Treatment of Egyptian Patients with HCV Genotype IV with Simeprevir and Sofosbuvir. Health, 8, pp.780-786.

Kwo P., Gitlin N.,and Nahass R., (2016). Simeprevir plus sofosbuvir (12 and 8 weeks) in hepatitis $\mathrm{C}$ virus genotype 1-infected patients without cirrhosis: OPTIMIST1, a phase 3, randomized study. Hepatology (Baltimore, Md.), 64(2), pp.37080 .

Lawitz E., Diago M., and Roberts S., (2016). Simeprevir plus sofosbuvir in patients with chronic hepatitis $C$ virus genotype 1 infection and cirrhosis: A phase 3 study (OPTIMIST-2). Hepatology (Baltimore, Md.), 64(2), pp.360-9. 
Press D., (2015). Efficacy and safety of a herbal mixture ( Viron ${ }^{\circledR}$ tablets ) in the treatment of patients with chronic hepatitis $\mathrm{C}$ virus infection : a prospective, randomized, open-label, proof-of-concept study. pp.799-804.

Sulkowski M., Cooper C., and Jacobson I., (2014). Daclatasvir plus sofosbuvir for previously treated or untreated chronic HCV infection. The New England journal of medicine, 370(3), pp.211-21.

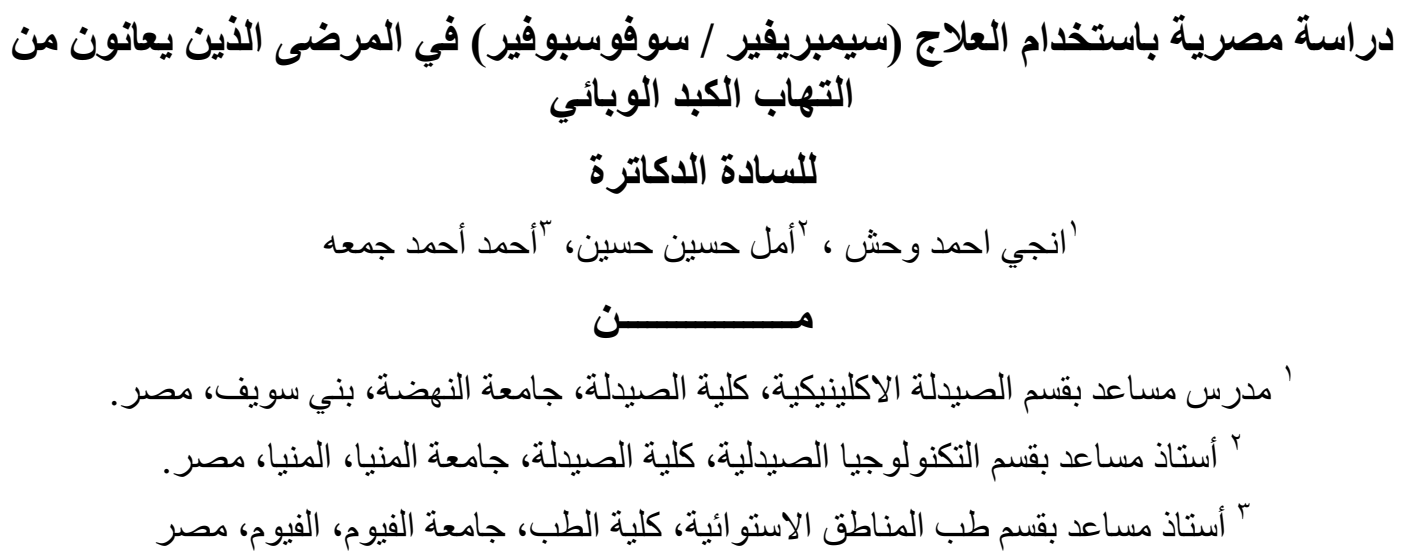

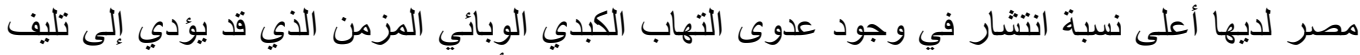

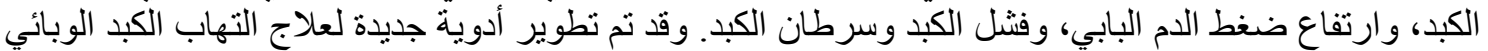

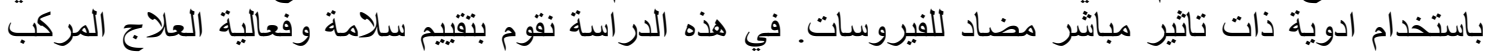

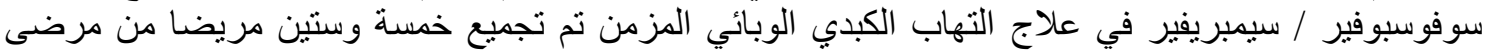

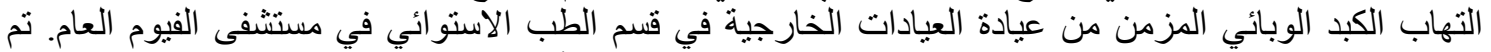

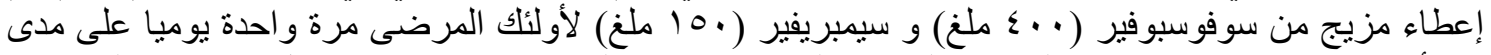

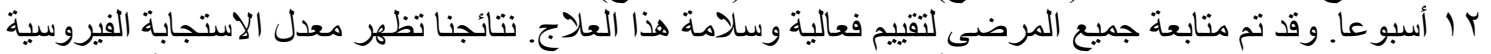

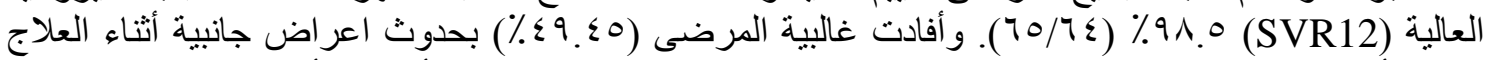

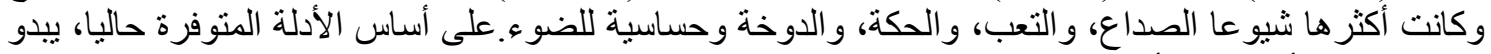

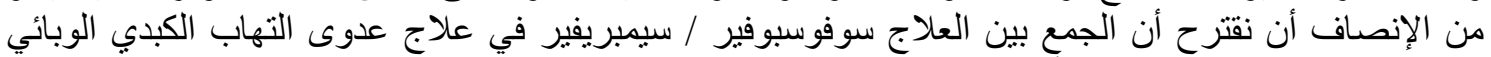

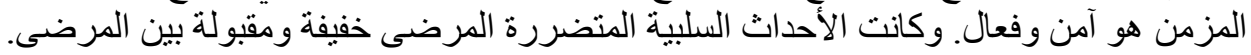

